\title{
Gerhard Armanski \\ Überlegungen zum Verhältnis von \\ Mensch, Natur und Gesellschaft
}

Lenz sagte, daß der Geist des Wassers über ihn gekommen sei, daß er dann etwas von seinem eigentümlichen Sein empfunden hätte. Er fuhr weiter fort: Die einfachste, reinste Natur hinge am nächsten mit der elementarischen zusammen; je feiner der Mensch geistig fühlt und lebt, um so abgestumpfter würde dieser elementarische Sinn; er halte ihn nicht für einen hohen Zustand, er sei nicht selbständig genug, aber er meine, es müsse ein unendliches Wonnégefühl sein, so von dem eigentümlichen Leben jeder Form berührt zu werden, für Gesteine, Metalle, Wasser und Pflanzen eine Seele zu haben, so traumartig jedes Wesen in der Natur in sich aufzunehmen, wie die Blumen mit dem $\mathrm{Zu}$ - und Abnehmen des Mondes die Luft.

Georg Büchner

Lenz

Die Tätigkeit und der Genuß, wie ihrem Inhalt, sind auch der Existenzweise nach gesellschaftlich, gesellschaftliche Tätigkeit und gesellschaftlicher Genuß. Das menschliche Wesen der Natur ist erst da für den gesellschaftlichen Menschen; denn erst hier ist sie für ihn da als Band mit den Menschen, als Dasein seiner für den andren und des andren für ihn, wie als Lebenselement der menschlichen Wirklichkeit, erst hier ist sie da als Grundlage seines eignen menschlichen Daseins. Erst hier ist zum sein natürliches Dasein sein menschliches Dasein und die Natur für ihn zum Menschen geworden. Also die Gesellschaft ist die vollendete Wesenseinheit des Menschen mit der Natur, die wahre Resurrektion der Natur, der durchgeführte Naturalismus des Menschen und der durchgeführte Humanismus der Natur.

Karl Marx

Ökonomisch-philosophische Manuskripte

Die folgenden Thesen versuchen, die historische Dialektik des Verhältnisses von Mensch und Natur als eine solche der rohen, unbegriffenen Einheit über die feindliche Trennung bis zur möglichen und notwendigen bewußten Wiederherstellung der Einheit auf höherer Ebene zu umreißen.

Der Rückgriff auf die Geschichte des Naturbewußtseins läßt seine gegenwärtige Form als nur eine denkbare (und daher auch abschaffbare) deutlicher hervortreten. In der archaischen Frühzeit des Verhältnisses von Mensch und Natur ist in embryonaler Form das Versprechen der Versöhnung mit der Natur enthalten, das erst nach der langen Periode der Klassengesellschaften auf einer neuen Stufe der gesellschaftlichen Entwicklung, in der sozialistischen Zukunft, realisiert werden kann.

Die klassengesellschaftliche Nutzung und Unterwerfung der Natur in ihrer höchsten bürgerlichen Form ist in eine Sackgasse geraten. Der unmittelbaren Abhängigkeit von der Natur weitgehend entronnen, erscheint das nunmehr herrscherliche Verhältnis des Menschen zu ihr seinerseits als unkontrollierte Naturgewalt, welche die Fortexistenz des Menschen selbst in Frage stellt. Die Erde ist vom Griff einer 
maßlos aneignenden Gesellschaft gezeichnet, vergiftet und vielerorts ungastlich geworden.

In ökologischen Katastrophen und neuen Krankheiten im menschlichen Körper wehrt sich die Natur. Auch unter den Menschen wird Widerstand wach, Sie kritisieren den Wachstumsfetisch, wollen den weiteren Raubbau an der Erde verhindern und die Reste unbehelligter Natur schützen. Eine neue, brüderliche Beziehung zur Natur dämmert auf.

Der Boden, auf dem das geschieht, ist noch derselbe, welcher das Übel hervorgebracht hat, das man vertilgen will. Die Krankheit scheint durch allerlei Rezepte und Kuren heilbar. Weil es um eine neue Aufgabe geht, sind die vorgeschlagenen Lösungsformen unreif und buntscheckig. Auch in Bezug auf die Natur stecken die bewußt werdenden und handelnden Menschen im Gewand der Denkformen einer bestimmten Gesellschaft. So erscheint die drohende Katastrophe als blindes Verhäng. $\mathrm{nis}, \mathrm{zu}$ dessen Erklärung allenfalls negativ anthropologische Gründe, nicht aber gesellschaftlich-geschichtliche herangezogen werden. Selbst die Schutz- und Rettungsvorschläge sind weithin vom gleichen quantifizierenden Kosten-Nutzen-Denken geprägt wie der Vorgang, der sie verursacht hat. Auf die Naturverwüstung antwortet die andere Seite der bürgerlichen Seele mit Rückzug, lnnerlichkeit, Einzelmenschlichkeit. Während im Erleben der Naturzerstörung vielfach die Vorstellung von der bürgerlichen Welt als der besten aller Welten birst, sind die Umrisse eines neuen Bildes von Mensch, Natur und Gesellschaft noch vage.

Was ist Natur? Außerer und innerer, materieller und geistiger Lebensprozeß vor und außerhalb des unmittelbaren Geltungsbereichs der Gesellschaft und ihrer Gesetze. In gewaltigen Zeiträumen aus kosmischen Vorgängen entstanden, ohne menschliches Zutun, ist sie seit dem Auftreten der ersten menschenähnlichen Gesellungen vor mehr als zwei Millionen Jahren nicht mehr autonom. Aus dem Blickwinkel der Naturgeschichte mögen die ersten Menschen als eine Laune der Natur und erbärmlich ausgestattete Lebewesen erschienen sein. Und doch haben sie sich in planvoller Arbeit über alle anderen Kreaturen erhoben und die Erde unterworfen. Die Natur ging in die geschichtlich-gesellschaftliche Tätigkeit des Menschen ein aber nicht in ihr auf. Sie erscheint immer weniger als sich selbst ïberlassene, unberührte. Sich im mitteleuropäischen Wald, in der Natur" im Gegensatz zur Gesellschaft zu wähnen, mutet angesichts von dessen fast vollkommener Eigenschaft, Produkt der menschlichen Kultur zu sein, platt und mystifizierend an. Was wir vor und in uns haben, ist Natur gesellschaftlicher Qualität, die freilich auch immer eigengesetzliche, ungezähmte Anteile mit sich führt. ln diesem Sinne ist sie nach wie vor die Basis menschlichen Lebens.

Es ist widersprüchliche Einheit: der Mensch unterwirft sich in seiner Arbeit die Natur, bleibt aber auch und gerade in ihrer gewandelten (selbst wieder naturmächtig auftretenden) Form an sie gebunden. Der Mensch ist Meister der Natur und noch immer Naturwesen. Tätiges und planendes Subjekt konnte er nur werden, indem er sich außerhalb der Natur und gegen sie stellte, sie damit objektivierte. Die Veränderung der Natur war seine eigene Veränderung als gesellschaftliches Wesen. lnnerhalb der klassengesellschaftlichen Geschichte verlief dieser Gegensatz durchaus 
als feindlicher - wilde Natur versus Unterjochung durch Wissen und Willen. So gang und gäbe ist das heute geworden, daß die Natur immer schon als das Andere gilt, sei es in der oben bezeichneten platten Form, sei es auch darin, daß die heutigen Umweltschützer als Vorboten eines neuen Verhältnisses zu ihr dennoch von Umwelt reden. Selbst in der Sprache macht sich das bewußtlose Auseinandertreten von Natur und Mensch geltend.

Geschichte ist die des Menschen und der Natur zugleich und in einem, in sich selbstzerrissen und widersprüchlich. Die Gesellschaft erhebt sich auf der Natur. lhre Geschichte wird zu deren Bewegungsform und -raum. ,Die Natur ist der unorganische Leib des Menschen, nämlich die Natur, soweit sie nicht selbst menschlicher Körper ist. Der Mensch lebt von der Natur, heißt: Die Natur ist sein Leib, mit dem er in beständigem Prozeß bleiben muß, um nicht zu sterben" (1). In der Bearbeitung der Natur erst wird der Mensch zum Gattungswesen, vergegenständlicht, verdoppelt er sich. In der Gesellschaft des Privateigentums aber geschieht diese Arbeit zum Zwecke der Vermehrung fremden Reichtums. Die entfremdete Arbeit entfremdet dem Menschen die Natur und sich selbst als tätiges Wesen von seiner Gattung und ,,verwandelt seinen Vorzug vor dem Tier in den Nachteil, daß sein unorganischer Leib, die Natur, ihm entzogen wird ${ }^{66}(2)$. Sie ,entfremdet dem Menschen seinen Leib, wie die Natur außer ihm, wie sein geistiges Wesen, sein menschliches Wesen“. Im Verhältnis des Mannes zur Frau zeigt sich schlagend das Verhältnis des Menschen zu sich selbst und zur Natur unter den Bedingungen der Entfremdung: Raub (3). Haben und Aneignen ist das Gesetz des Privateigentums.

Die Feindschaft zwischen Mensch und Natur wird es so lange geben, wie die aufs Privateigentum gegrïndete Klassengesellschaft existiert. Der Kapitalismus als höchste Form der Klassenspaltung muß auch diese Feindschaft aufs heftigste zuspitzen. In seinen antagonistischen Klassenformen und der schier grenzenlosen Auspowerung der Natur hat er die Grundlage eines befreiten, menschlichen, reichen Lebens jenseits der Klassengesellschaft geschaffen. Gerade auch für das Verhältnis zur Natur gilt, daß die Entwicklung der Produktivkräfte in den gegensätzlichen Formen des Raubs und gesellschaftlich schöpferischer Potenz voranschreitet. Je höher der Entwicklungsgrad, desto schneidender auch der Gegensatz. Umso dringlicher ist die Forderung, das destruktive Umkippen dieser Entwicklung zu verhindern und selbst der Gefahr einer solchen durch die Überwindung der Klassengesellschaft den Boden zu entziehen. Unmittelbares Handlungsgebot und Perspektivsicherheit sind gleichermaßen dringlich. Erst der ,Kommunismus ist als vollendeter Naturalismus = Humanismus, als vollendeter Humanismus = Naturalismus, er ist die wahrhafte Auflösung des Widerstreits zwischen dem Menschen mit der Natur und mit dem Menschen, die wahre Auflösung des Streits zwischen Existenz und Wesen, zwischen Vergegenständlichung und Selbstbestätigung, zwischen Freiheit und Notwendigkeit, zwischen In-

1 Karl Marx, Ökonomisch-philosophische Manuskripte, 1844. Marx/Engels Werke (MEW), Berlin (DDR), Ergänzungsband 1, S. $516 \mathrm{f}$.

$2 \quad$ A.a.O., S. 517

3 Vgl, a.a.O., S. 535 
dividuum und Gattung. Er ist das aufgelöste Rätsel der Geschichte und weiß sich als diese Lösung" (4).

Die Alten (4a), vor der Klassengesellschaft und in ihren frühen Phasen, sahen die Natur nicht als äußeren Gegenstand an. lhr Leben beruhte unmittelbar auf ihr. Die Menschen waren ein ins Weltganze eingefügter Teil desselben; die Natur wiederum reichte weit in ihr Sinnen und Trachten hinein. Die Erde war die Spenderin des Lebens, der Mond ihr zyklisches Zeitmaß. Als Muttergöttinnen schufen und krönten sie die Welt. Die Gesellschaft nach ihrem Bilde war matristisch, der Fruchtbarkeit der Erde und des Schoßes anheimgegeben.

Schöpfungsmythen, Märchen, religiöse Kulte widerspiegeln die Ungeschiedenheit von Mensch und Natur. ,Tiere agieren wie Menschen, Menschen verwandeln sich in Tiere und umgekehrt, die Helden verfügen über unmenschliche Kräfte, und die Gegenstände besitzen wunderbare Eigenschaften ... In die Märchenhandlung ist die gesamte Natur einbezogen als vermenschlichte Natur, indem der Mensch sich selbst, seine eigene soziale Organisation (zunächst die Stammesorganisation, später ... die Klassengegensätze) in die Natur hineinprojiziert bzw. indem er die in der Natur enthaltenen potentiellen Möglichkeiten für den Menschen im Märchen bereits erschließt zu einer Zeit, da der Bereich der durch den Menschen erkannten GesetzmäBigkeiten in der Natur noch außerordentlich begrenzt war und breiten Raum für phantastische Vorstellungen ließ " (5). Natur und Mensch sind eng miteinander verwoben. Der Mensch fühlt sich allmächtig (als Mitglied des brüderlichen Welthauses) und allohnmächtig (dem rätselhaften Agieren der Natur unterworfen) zugleich. Sein Empfinden und Weltsinn steht den Objekten nicht gegenüber, lagert sich an die Objekte an, verschmilzt gar mit ihnen. Die Grenzen zwischen Subjekt und Objekt sind verschwommen. Das Ich ist unentfaltet, in ursprüngliche und existentielle Kollektivität eingebunden und hinterläßt keine Spur. ,,Was ist das Leben des Menschen? Der Schatten eines Vogels über der Prärie, der in der sinkenden Sonne vergeht" (6).

Unendlich und mächtig erschien die Natur. Sich in sie einzufügen war Grundbedingung menschlichen Glücks. Außerhalb der natürlichen Schöpfung konnte der Mensch, selbst ein Teil von ihr, nicht leben. Der Mensch stand im engen, lebenswichtigen Verhältnis zum Tier. Die in den Höhlenmalereien zaubrisch gebannte Jagd-

$4 \quad$ A.a.O., S. 536

4a Diese Zusammenfassung vorkapitalistischer Gesellschaftsformen erscheint sowohl durch die von mir gewählte Art der Darstellung wie durch die Tatsache gerechtfertigt, daß die qualitativ entscheidende Abhebung von der Naturgrundlage des Lebens mit der kapitalistischen Klassengesellschaft erfolgt. Ihre erheblichen Unterschiede untereinander sollen deswegen keineswegs verwischt werden.

5 J. Klagge, Mensch und Natur im russischen Märchen, in: Bruno Schrage (Hrsg.), Das Naturproblem in der realistischen Literatur, 1. Teil, Rostock 1975, S. 69. Vgl. August Nitschke, Sozjale Ordnungen im Spiegel der Märchen, 2 Bde., Stuttgart 1976/77 Kiowa-Sprichwort 
beute war nicht bloß Nahrungsmittel, sondern Bruderwesen, dessen Tötung man erflehte und zugleich rituell auszusöhnen trachtete. Der Umgang mit der Natur war vertraut, ehrfürchtig, ihren Bestand kaum gefährdend. Wie kann man das Gras der Prärie schneiden und Bergbau betreiben - kann man denn seiner Mutter das Haar abschneiden und ihr die Knochen aus dem Lei nehmen? (7) Die Kehrseite und Grundlage dieser Ehrfurcht ist die vollkommene Angewiesenheit auf die Natur, der man auf Gedeih und (oft genug) Verderb ausgeliefert war. Die Unterschiede in Verhalten und Einstellung werden gut bei der Büffeljagd sichtbar. Den einen war sie umfassende materielle Lebensgrundlage, die weit in die psychosoziale Struktur hineinreichte, den anderen schließlich bloß Betätigung eines verschwenderischen Aneignungs- und Tötungsrausches.

Die frühen Menschen lebten in der „Scheu vor der Störung der Weltsymmetrie" (8), im Anschmiegen an die Wirkung des Kosmos, in der Antwort an den Himmel (9). Entsprechend war auch die soziale Struktur als kosmisch gestiftet angesehen, unwandelbar und autoritär, und suchte in Ritus und Alltag die Einheit mit ihrem Schöpfungsgrund. „Die Gemeinschaft des Stammes oder Dorfes wiederholt die Gestalt des Alls" (10). Stammesmythen begründeten und bewahrten diese Einheit, weihten die alläglichen und außergewöhnlichen Verrichtungen der Menschen. Rituale bekräftigten, Amulette und Körperornamente symbolisierten sie. Tierclanmäßige Stammesorganisation, Totempfähle zeigen diese unauflöslich enge Verwobenheit von Natur und Gesellschaft genauso an, wie das durch Beobachtung und bildhaftes (gestalt therapeutisches) Erkennen gewonnene erstaunliche Naturwissen etwa in der Heilkunde, ausgeübt durch den Schamanen und dessen soziale Stellung begründenḍ. Seine Heilkunst besteht im Grunde im ,Einschwingen des Menschen in das All" (11), in der Wiederherstellung eines zerrissenen natürlichen und sozialen Zusammenhangs. Beispielhaft hierfür sind die ungeheuer verwickelten Sandmalereien der Navajos.

Bei einem geringen Entwicklungsgrad der Arbeitsmittel und der sozialen Organisation erklären durch Generationen angehäuftes praktisches Wissen, nicht Wissenschaft, Anschauung und Bild, nicht der Begriff, die Welt und die inneren Lebenszusammenhänge. Materiell gründet das auf der engen Abhängigkeit von den Naturbedingungen des Lebens und ihr Fortdauern in der frühen jagdmäßig-agrarisch geprägten Produktionsweise.

Die frühe Periode der Menschheit war durch archaische Naturverbundenheit (und darin auch Geborgenheit) gekennzeichnet. Eine Insel der Seligen war sie nicht. Sie war Kampf und Demut gegenüber einer unberechenbaren Naturmacht, die man nicht beherrschen, sondern nur versöhnen konnte. Beides lag in der noch nicht auseinandergefalteten Beziehung von Mensch und Natur und beides mußte sich durch die anhebende zivilisatorische Entwicklung verändern: Indem sich die Menschheit

7 Rede des Häuptlings Smohalla

8 Werner Müller, Indianische Welterfahrung, Stuttgart 1976, S. 46

9 Wie es die altchinesische Religion sah.

10 Werner Müller, a.a.O., S. 70

11 A.a.O., S. 40 
in ihrer klassengesellschaftlichen Epoche von ihrem sozialen und technischen Naturzustand emanzipierte, sich dabei selbst als Subjekt setzte, trat sie aus dem primitiven gesellschaftlichen Gehäuse, aus Naturfurcht und Naturwärme, heraus. Es war das Ende der Magie. Die ursprüngliche Einheit barst. Die biblische Legende von der Vertreibung aus dem Paradies drückt diesen Vorgang aus. Während sie den ursprünglichen Zustand zum Garten Eden verklärt, verheißt sie beim Aufbruch aus ihm einen mühseligen und plagevollen Weg der Menschwerdung. In der sehnsüchtigen Erinnerung liegen aber auch nach vorn gerichtete Hoffnungselemente auf ein besseres und friedliches Leben, auf ein neues Paradies als Ende und Erfüllung der Geschichte. Nicht umsonst ist die Geschichte voll von Experimenten und Traktaten zu diesem Thema.

Der widersprüchliche Charakter des archaischen Erbes ist gegen Versuche festzuhalten, ihn idealisierend-reaktionär oder fortschrittsblind auseinanderzuschneiden. Dem einen handelt es sich um Unschuld, die der sündigen Zeit mahnend als Spiegel entgegengehalten wird. Seine Stimme, ,weit entfernt von selbstbeweihräuchernden Redewendungen wie ,Krone der Schöpfung" oder ,Beherscher der Natur" " (12), tönt in einem recht modischen Kulturpessimismus, den die jüngste Welle von Naturzerstörung in der Nachkriegszeit hervorgerufen hat. Der andere sieht bloß die ande. re Seite, die Befreiung von der blinden Naturmacht, die zivilisatorische Herrlichkeit von Vernunft und Technik und hat keine Augen für den hierfir in der klassengesellschaftlichen Entwicklung gerichteten Preis der Verstimmelung und Verkümmerung der äußeren und inneren Natur. Von diesem Überschwang der bürgerlichen Fortschrittsphilosophie ist auch Marx nicht frei (12a) und seine realsozialistischen Erben schon gar nicht. Er spricht von der ,Natur, die den Menschen anfangs als eine durchaus fremde, allmächtige und unangreifbare Macht gegeniiberterit, zu der sich die Menschen rein tierisch verhalten, von der sie sich imponieren lassen wie das Vieh; und also ein rein tierisches Bewußtsein der Natur (Natureligion) ... Diese Naturreligion oder dies bestimmte Verhalten zur Natur ist beding turch die Gesellschaftsform und umgekehrt. Hier wie überall tritt die ldentität von Natur und Mensch auch so hervor, daß das bornierte Verhalten der Menschen zur Natur ihr borniertes Verhalten zueinander, und ihr borniertes Verhalten zueinander ihr bonniertes Verhältnis zur Natur bedingt, eben weil die Natur noch kaum geschichtich modifiziert ist" (13). Was die Menschheit zu welchem Zweck und Ende verließ, blebibt unausgedrückt.

Die geschichtliche Modifikation der Natur, ihre Unterwerfung, eruchtbarmachung und Zerstörung nämlich, beginnt in den fruhen Klassengesellschoten. Auf dem breiten agrarisch-jagdmäßigen Unterbau, der entweder auf den Besingl der Natur achte-

12 Kurt Bliiche1, Untergang der Tiere, Stuttgat $1976, \mathrm{~S} .91$

12a Vgl. Hellmuth Scheh1, Vor uns die Sintfit?, Okologie, Marxismus und die herrschende Zukunftsgläubigkeit, Berlin (W) 1977

13 Karl Marx/Friedrich Engels, Deutsche Ideologie, 1845/46, MEW 3, 5. 31 
te oder ihn noch gar nicht zu bedrohen imstande war, erwachsen zerstörerische Aktionen vor allem dort, wo sich die Gesellschaft von der natürlichen Reproduktion des unmittelbaren Lebens abhebt, in Militärbereich und Handelswesen (erste Bewegungsformen von Geld und Kapital): Xerxes läßt den Hellespont, den er überqueren will, mit Ruten züchtigen; Römer und Venezianer holzen lstrien ab.

Auch innerlich wird die Natur zum féindlichen Gegenüber. Jahrtausendelang arbeiten die monotheistischen Religionen - Juden- und Christentum an der Spitze - daran, die zivilisatorische Leistung des modernen, produktiven lndividuums zu vollbringen, indem sie die stammesgesellschaftliche Triebstruktur der „Natürlichkeit" (Leben in den Tag hinein, Regierung der Sinne) unterjochen bzw. zu unterjochen trachten. Gelungen ist das bis heute nicht ganz. Das Christentum ist das Manifest der Unterwerfung der inneren und äußeren Natur unter das patriarchale (Über) Ich, Gewissen, Herrschertum, Individuum - eine Art Produktionsanweisung zur Bildung des menschlichen historischen Subjekts. Und der mosaische Gott hatte geboten: „Macht Euch die Erde untertan!“ und verheißen: „Seid fruchtbar und mehret euch und erfüllet die Erde. Euer Furcht und Schrecken sei über allen Tieren auf Erden, über allen Vögeln unter dem Himmel und über alles, was auf dem Erdboden kreucht, und alle Fische im Meer seien in eure Hände gegeben. Alles, was sich reget und lebet, das sei eure Speise. Wie das grüne Kraut habe ich euch alles gegeben" (14).

Während die antiken Mysterien als letzte (europäische) Form der kultischen Vereinigung von Mensch und Natur in matristischer Tradition verfallen, breitet sich das jüdisch-christliche herrscherliche Verhältnis gegenüber den natürlichen Sinnen und Reichtümern aus. Die Zeit der großen kosmo- und theogonischen Naturdichtungen und Weltdeutungen geht vorüber. Frühe Naturwissenschaft, die sich des Begriffs, der Abstraktion, der Verallgemeinerung bedient, die nach den Gesetzen hinter den Erscheinungen der Natur sucht, dringt vor. Das lndividuum stürzt ins Dasein, der Kreis der Zeit bricht. Der Hellenismus ist das große Wort dieser philosophischen Zeitenwende.

Schon damals ruft die abwendende Unterwerfung der Natur Reflektionen über das Verlorene hervor, Versuche, heiter-wehmütige, künstliche und vergebliche jedenfalls, sich mit ihr wiederzuvereinigen. Spielerisch-schwärmerische Naturbegeisterung (Anakreontik) signalisiert nur die Trennung und wird sie fortan begleiten. Im praktischen Handgemenge mit einer zusehends entseelten Natur löst sich die abstrakte christliche Naturauffassung (Gottes Geschenk, Fluch und Sünde) und das neue herrscherliche Kunstideal ,Natur "Non ihrem Gegenstand ab. So ist zum Beispiel die spätere, Naturdichtung des Minnesangs ... nicht Abbild der Wirklichkeit wie sie ist, sondern Bild einer schönern angestrebten Welt (in die der ritterliche Mensch mit all seinem Empfinden, Denken und Vorstellen durch tausendfache Fäden hineingesponnen ist) "(15). Die höfische Asthetisierung der Natur legt aber auch Zeugnis $a b$ von dem noch lange nicht ausgefochtenen Kampf mit ihr und ihren Dämonen (siehe die Naturfratzen an den mittelalterlichen Kathedralen). Sie , ,könnte

14 1. Mose $9,1-3$

15 Ludwig Schneider, Die Naturdichtung des deutschen Minnesangs, Berlin 1938, S. 29 
wohl in dieser Empfindung des Dämonischen zutiefst wurzeln, das, halbgöttlich, zugleich geflohen und geliebt wird, insofern nämlich, als durch die Versprachlichung und Stilisierung das Dämonische gemildert und verwandelt wird“ (16).

Die (verklärende und treibende) Erinnerung an einen paradiesischen Urzustand, die desto weniger mit ihrem widersprüchlichen, rauhen und bergenden, Urzustand sich deckt, je weiter sie von ihm entfernt ist, zieht tiefe Spuren im mensch(heit)lichen Gedächtnis. Nicht zufällig tritt der emphatische Ruf Rousseaus „Zurück zur Natur!" (natürlich auch und vor allem als naturrechtliche Basis der Kritik an überholten feudalistischen Zuständen) historisch gerade dann auf, als mit dem bürgerlichen Zeitalter die letzte Phase der Ablösung von der Natur und ihrer Nutzbarmachung/Zerstörung heraufzieht. Ironischerweise sind es (in den Fabeln Lafontaines) Tiere selbst, die in Bürgergestalt auftreten - und später von der Zivilisation eben jener Bürgergestalt an den Rand ủer Ausrottung gedrängt werden. Ähnliche Paradoxien kennzeichnen oft die naturkundlichen und geographischen Entdeckungsreisenden während der Aufklärungszeit.

Bevor die Natur untergeht, dient(e) sie nicht nur der frühbürgerlichen Gesellschaftskritik als Folie (wie übrigens auch späteren kulturpessimistischen Philosophien, etwa der Spenglers), sie wird auch zum Rückzugsgehäuse des sich nach hinten und weltabgewandt versenkenden bürgerlichen Gemüts, der zarten Seele der bürgerlichen Kraft- und Herrschernatur. Die Romane und Volkslieder der Romantik zeugen davon. In dieser verdrehten Form wird die Natur seelisch ins bürgerliche Ich eingelagert. Die Verlegung der persönlichen Sehnsüchte und Schmerzen in die Natur als menschliche Symbolwelt ist bis heute Bestandteil der bürgerlichen lchformation (vor allem in der Jugendzeit) geblieben. ,Mein Herz sieht an den Himmel gemalt sein eigen Bild. - Es ist nichts als der Winter, der Winter kalt und wild! / Schnee, du weißt von meinem Sehnen; sag, wohin doch geht dein Lauf?" (17) Oder: „So heiß und stumm, so trübe und sternlos war die Nacht, so ganz, wie unsre Liebe, zu Tränen nur gemacht" (18). Auf eine verquere Weise gibt es hier immerhin noch das Zwiegespräch mit der Natur.

\section{1}

In der Epoche des bürgerlichen Industrialismus verselbständigt sich der gesellschaftliche Lebensprozeß am weitesten von seinen natürlichen Grundlagen. Technologisch und naturwissenschaftlich betriebene Ausbeutung der Natur und des Menschen ist der Kern einer Produktionsweise, für die nur der abstrakte gesellschaftliche Reichtum und seine Vermehrung zählt. „Das unendliche Kreisen (der frühen Gesellschaften, G.A.) geht über in einen unendlichen, ziellosen Progreß, dem die individualund klassenegoistisch zersplitterte Gesellschaft ähnlich ohnmächtig gegenübersteht wie vorher die älteren Gesellschaften der nicht oder nur halb gezähmten ersten Na-

16 A.a.O., S. 115

17 Franz Schubert, Eine Winterreise

18 Nikolaus Lenau 
tur $^{\text {" }}$ (19). Dies ist der klassengesellschaftliche Preis für den geschichtlichen Austritt aus Naturverfallenheit und primitiver Schicksalsblindheit: die Befreiung von der Natur erscheint in der widersprüchlichen Form ihrer Unterwerfung und Zerstörung. In dieser Form wird nun die Gesellschaft selbst zur naturwüchsigen Schranke, vor der sich die historische Dialektik der Naturbeherrschung staut und destruktiv wendet, zur nächsten Stufe der Versöhnung von Mensch und Natur nicht fortgehen kann. Wenn die Geschichte eine solche des Menschen und der Natur ist, hierin eine gegensätzliche Einheit darstellt; wenn sich die Menschen in ihrem klassengesellschaftlichen Fortschritt der Natur zu bemächtigen hatten, so folgt nun, daß erst die Abschaffung dieser klassengesellschaftlichen Form des Fortschritts, der Herrschaft des Menschen über den Menschen einen befreit-gleichberechtigten Umgang mit der Natur ermöglicht.

Mit der Herausbildung der kapitalistischen Klassengesellschaft und des (männlichen) bürgerlichen Individuums seit dem späten Mittelalter geht die eines Eroberungs- und Gewaltverhaltens gegenüber dem Weltball - als Kolonie der heraufkommenden Rasse und Kultur - einher, seinen Tieren, Pflanzen, Mineralien, auch und vor allem Völkern („Naturvölkern“) gegenüber. Neu-gier als Vorbote und Begleiter von Habgier durchzittert die Entdeckungsfahrten und Reiseberichte von Kolumbus bis Stanley, kaum aber Respekt. In einem nie gekannten Maß wird die Natur gebändigt und genutzt. Der Stoffwechsel des arbeitenden Menschen mit der Natur treibt auf sich rapide erweiternder technologischer Grundlage ungeahnte Reichtümer hervor. Aber er erfolgt in bestimmten, der kapitalistischen Herrschaft und Zwecksetzung adäquaten Formen. So sehr sich die sprunghafte Steigerung der Produktivität der Arbeit Naturbedingungen verdankt, so wenig werden diese gehegt, vielmehr schrankenlos bis an die Grenze ihrer Vernichtung ausgebeutet. Auf der Grundlage eines historisch programmierten Wildbeutertums tritt die aufs höchste gesteigerte kapitalistische Naturaneignung als schließlich tendenziell selbstzerstörerisch auf wenn ihr nicht gewehrt wird. Dies ist das Doppelgesicht der bürgerlichen Entwicklung, auch bezüglich der Natur: ,Jeder Fortschritt der kapitalistischen Agrikultur ist nicht nur ein Fortschritt in der Kunst, den Arbeiter, sondern zugleich in der Kunst, den Boden zu berauben, jeder Fortschritt in Steigerung seiner Fruchtbarkeit für eine gegebne Zeitfrist zugleich ein Fortschritt im Ruin der dauernden Quellen dieser Fruchtbarkeit ... Die kapitalistische Produktion entwickelte daher nur die Technik und Kombination des gesellschaftlichen Produktionsprozesses, indem sie zugleich die Springquellen alles Reichtums untergräbt: die Erde und den Arbeiter ${ }^{\text {“6 }}$ (20).

Das Aneignungs- und Vergewaltigungsverhältnis zur Natur (jeder Vorstoß in letzte unbekannte Regionen, z.B. das Antarktiswasser, wird wie eine Entjungferung gepriesen), regional und kulturell unterschiedlich, aber in jedem Individuum und allen Handlungen der bürgerlichen Welt präsent, drückt ihre Herabsetzung zur bloßen

19 Reinhard Maurer, Natur als Problem der Geschichte, in: Kurt Hübner/Albert Menne (Hrsg.), Natur und Geschichte, X. Deutscher Kongreß für Philosophie 1972, Hamburg 1973, S. 130

Karl Marx, Das Kapital, Band I, MEW 23, S. 529 f,; vgl. auch Band III, MEW 25, S. 821 
Grundlage des sich verwertenden Werts aus. An die Stelle der alten Naturzyklen ist das schiere Forttreiben linearer Endlosigkeit getreten und ein mit ihr einhergehender quantifizierender Zeitbegriff.

Die Zeit des archaischen Bündnisses des Menschen mit der Natur ist dahin. Die zehntausendjährige Arbeits- und Lebensgemeinschaft von Mensch und Haustier löst sich auf. Die Menschheitsgeschichte bedeutet auch fortschreitende Loslösung vom Tier - vom Jäger des Menschen wird es zu seiner Jagdbeute, dann zum Arbeitsund Hausgenossen, schließlich zum industriellen Rohstoff, zum Luxusgegenstand oder Relikt, an den Rand der Weltbühne gedrängt jedenfalls. Die Geschichte der Kunst und des Rechts widerspiegeln sehr deutlich diese Kämpfe und Machtverschiebungen - von den ehrfürchtigen Höhlenmalereien über die Löwenjagd der Pharaonen bis zum Herrenreiter, vom Tier als Bruder zum Tier als vernunftlosem Rechtsobjekt. Das hauptsächliche Motiv heutiger Beschäftigung mit dem Tier, selbst in der Zoologie, ist anthropozentrisch (20a): das Tier dient zur Befriedigung menschlicher Bedürfnisse, ist zum Menschersatz (z.B. für alte Menschen), zum Sport- oder Jagdgegenstand geworden. Neulich ist gar die Züchtung eines Hundes, der nicht bellt, gelungen - zynische Fußnote auf die (natur)historische Niederlage des Tieres gegenüber dem Menschen.

Die naturreligiösen Kulte, wo sie in den Kolonien noch existierten (z.B. Sonnentanz der Sioux) wurden von den bürgerlichen Weißen verhöhnt und verboten. In ihren eigenen Breiten sterben die alten Jahreszeit- und Naturfeste (z.B. Winteraustreibung, Sommersonnenwende) aus. Bisweilen werden sie künstlich, in erinnernder Sehnsucht und Folklore wiederbelebt. Die unterdrückte Natur tritt gedemütigt in Zoo und Zirkus als kolonialistische Kulturprodukte auf. Bisweilen wird sie auch museal geschützt. Ansonsten ist sie schierer Gegenstand und bloßes Arbeitsmaterial geworden. Schon die Sprache drückt das aus: Rohstoff, Jagdtourismus, Tierhandel, Nutzfläche, Gelände, Grünland, Holzindustrie usw.

Die Unterwerfung ist den Menschen aber nicht recht geheuer. Noch immer sind Dämonisches und mögliche Rache in der Natur. Die genüßlich-schaudemde Verarbeitung von Naturkatastrophen zeigt das ebenso an wie filmgewordene Schuldgefühle gegenüber der gepeinigten Kreatur, gepaart mit Angst vor ihrem Aufstand (vgl. Filme wie Vögel, Ben, Orca, Squirm, Piranhas, Der weiße Hai) (21). Beides bezieht sich gewiß auch auf die unterdrückte Triebstruktur im bürgerlichen Ich, deren Herausbildung, wie wir gesehen haben, mit der Unterdrückung der äußeren Natur einhergegangen ist.

So wie der Tauschwert über den Gebrauchswert, die Quantität über die Qualität herrscht, so auch die Gesellschaft über die Natur, die Abstraktion über die Sinnlichkeit. Der Mensch, selber Teil der Natur, erscheint wiederum dem Kapital als natürliche Grundlage und Schranke zugleich seiner Selbstverwertung. Auf Ratio und Funktion ist er aber ausschließlich und dauerhaft, nicht zu reduzieren. Und zwar nicht nur, weil er die Reste unmittelbarer Emotionalität verteidigt, sondern weil

20a Vgl. Horst Stern, Mut zum Widerspruch, München 1974

21 Es ist sicher nicht zufälig, daß alle diese Filme aus den USA stammen, wo sich die Ero berung der Natur ganz besonders schnell und gewaltsam vollzog. 
Sinnlichkeit für bestimmte Verrichtungen in der bestehenden Gesellschaft ganz unentbehrlich ist (z.B. für Kindererziehung und Kreativität erfordernde Berufe). Gebrauchswert- und Sinnlichkeitsinseln sind also für die Formation der bürgerlichen inneren Natur durchaus funktional. In dieser Funktionalität gehen sie jedoch nicht auf. Sie schärfen die Sensibilität für die Unterdrückung der Sinne und stimulieren den Widerstand, womit sie objektiv und subjektiv die Kreise des Kapitals stören können. Freilich ist auch die kommerzielle Nutzung und Bewegungsform der Sinnlichkeit möglich.

Als wichtige Momente der kapitalistischen Formierung der inneren Natur, deren systematischer Zusammenhang hier nicht entwickelt werden kann, sind die Internalisierung der Arbeitsnormen ins Zeitbewußtsein, die tauschwertgemäße Umformung der Gegenstandswelt und die Entsinnlichung des menschlichen Körpers zu nennen (22). Alle diese (und andere) Momente zielen auf einen Menschen nach dem Bild der abstrakten, ,gleichgültigen“ und quantifizierbaren Ware, für die der lebendige, qualit ive Stoff nur noch Träger ihres ökonomischen Charakters ist.

Im gleichen Maße, wie konkret-nützliche Arbeit nur noch Träger abstrakt-gesellschaftlicher Arbeit wird, wo die Arbeitskräfte selbst zum Tauschwert, die Arbeit Außer-sich-Sein des Arbeiters geworden ist, ,gewinnt die Zeitform abstrakter Arbeit immer größeren Einfluß auf das konkrete Zeitbewußtsein: durch die taktmäßige Gleichförmigkeit der Produktion, die Notwendigkeit der Pünktlichkeit, die Quantifizierung der Arbeitsleistung, durch die Form der Entlohnung, durch die Dissoziierung von Produkt und Bedürfnis" (23). Zeit wird abstrakt und vermag weder Perspektive noch Sinn mehr zu stiften, eröffnet auch Zukunft nicht mehr. Diese kapitalistische Zeitstruktur kann die Person derart stören, daß ihre Anpassungsleistung und ihr Gebrauchswert fürs Kapital schwinden. Auch hier droht also das Kapital sich seiner eigenen Naturbasis zu berauben.

Die Produzenten sind nicht nur im Produktionsprozeß sich selbst entfremdet, ihnen ist auch ihr Produkt und schließlich die Konsumtion überhaupt entfremdet und entsinnlicht. In der Ästhetisierung der Warenwelt wirkt ein kapitalimmanenter Gegentrend hierzu. Die Entfremdung und die zerhackte Form von Objektbeziehungen durchs Privateigentum vermögen ebenfalls zur vollkommenen Bindungslosigkeit und zum Realitätsverlust zu führen und damit dysfunktional zu werden.

Schließlich kann der kapitalistische Produktionsprozeß nur mit disziplinierten, von sexuellen Triebzyklen und sinnlichen Wünschen weitgehend abgekoppelten Lohnarbeitern funktionieren. Die Geschichte der bürgerlichen Sexualunterdrückung (24) ist denn auch notwendige und qualvolle Vorbedingung und Begleiterscheinung der Plackerei der Lohnarbeit. In verdrängter Gestalt vermag Sexualität gar effizienzsteigernd zu wirken, was sie selbst lustärmer macht. Die bekannte ,repressive Ent-

\footnotetext{
22 Das Folgende nach einem Arbeitspapier von Niko Diemer und Gerd Klatt im Rahmen der Ernst Bloch-Tage 1978 mit dem Thema „Marxismus und Naturbeherrschung“"

23 Alfred Krovoza, Die Verinnerlichung der Normen abstrakter Arbeit und das Schicksal der Sinnlichkeit, in: C. Bezzel u.a., Das Unvermögen der Realität, Berlin 1974, S. 23

24 Vgl. Jos van Ussel, Sexualunterdrückung. Geschichte der Sexualfeindschaft, Neuauflage Gießen 1977
} 
sublimierung “ ändert an der Verdrängung und Verarmung von Sinnlichkeit und Körperbewußtsein nichts.

Die historische Notwendigkeit der rationalen Denkform und der Disziplinierung der Produzenten ist kaum zu bestreiten, es sei denn von einem rückwärtsgewandt-idealistischen Standpunkt aus. „Es ist eine der zivilisatorischen Seiten des Kapitals, daß es die Mehrarbeit in einer Weise und unter Bedingungen erzwingt, die der Entwicklung der Produktivkräfte, der gesellschaftlichen Verhältnisse und der Schöpfung der Elemente für eine höhere Neubildung vorteilhafter sind als unter den früheren Formen der Sklaverei, Leibeigenschaft usw." (25). Das Leiden einer endlosen Reihe von Generationen und Individuen, erzwungen durch Klassenherrschaft und Ausbeutung, ist die historische Voraussetzung der Überwindung derselben. Die Entwicklung und Vergesellschaftung der Natur, auch unter bürgerlichen Vorzeichen, hat erst die reelle Chance einer Humanisierung der Natur und einer Naturalisie rung des Menschen jenseits von Elend und Kampf gesetzt.

\section{IV}

Der Durchbruch zum Reich der Freiheit kommt aber nicht von selbst. Er kündigt sich darin an, daß die Produktivkräfte unter dem Joch der herrschenden Produktionsverhältnisse immer mehr zur Geißel anstatt zum Segen werden - die Maschinerie und Chemie ebenso wie der soziale Arbeitskörper und die anscheinend privaten Beziehungen. Er zeigt sich darin, daß sich die Sehnsucht nach anderen, menschlicheren und natürlicheren Lebensverhältnissen, erinnernd und vorwärtsdrängend selbst durch die bürgerlichen Panzer hindurch regt, sich entfalten und ausfliegen will. Die Bruchstellen im Panzer zu erkennen und dem Hervordrängenden ans Licht zu helfen - das ist die Aufgabe von Kommunisten, Sozialisten, radikalen Humanisten und Ökologen. Denn ,im Blick auf die entwickelte technische Zivilisation ... ist wohl nur noch in übergeschichtlicher, eschatologisch-theologischer Perspektive zu erwarten, daß in der bisherigen Weise fortschreitende Aufklärung und Naturbeherrschung zu einer Versöhnung von beherrschter Natur und beherrschendem Subjekt, und im Subjekt zu einer Versöhnung von Vernunft und Triebnatur führen würde" (26). Die bürgerliche Produktions- und Denkform droht in einer Sackgasse zu enden.

Die Natur leidet. In den letzten drei Jahrhunderten sind allein 500 Tier- und zahllose Pflanzenarten ausgerottet worden. Uralte Tiere wie der Alligator, die 120 Millionen Jahre überstanden haben, stehen vor dem Untergang. Die Basis der bloßen Begegnung mit belebter außermenschlicher Natur wird immer schmäler. So wird die folgende Warnung verständlich:

„Unsere fast vollständige Nichtachtung des Werts einer natürlichen Umwelt und unsere erfolgreichen Versuche, die Ordnung der Dinge drastisch zu verändern, wird letzten Endes für ein allumfassendes Unheil sorgen ... Seit der Jungsteinzeit ist der Mensch ungebrochen im Besitz jener Macht, sich die Erde untertan zu ma- 
chen. Während seiner Herrschaft hat er jedoch nahezu alle ökologischen Grundsätze durchbrochen, die für den Stoffkreislauf, die Isolation und Wechselbeziehung der Gemeinschaften sowie die natürliche Begrenzung der Bevölkerungszahlen (?!) gelten" (27).

Durch ihre scheinbare Plausibilität bricht hier voll der Zynismus und die negative Anthropologie (um das Böse im Menschen zu zähmen, könnte es eines starken Staates bedürfen) der bürgerlichen Kulturkritik durch. Sie ist ebenso moralisch wie hilflos: „Gier und Gift, Dummheit und Eitelkeit haben das Raumschiff Erde ins Trudeln gebracht" (28).

Grund für derartigen Pessimismus liegt genug vor (29), und in der Tat sind es auch die Vertreter jener Kulturkritik gewesen, die zuerst die Alarmtrommel gerührt haben. In den USA z.B. sollen die Todesfälle durch Pflanzenschutzmittel bereits diejenigen durch Verkehrsunfälle überschreiten. Die Luft wird rapide vergiftet, das Wasser verseucht. Die Zersetzung der Ozonschicht um den Erdball und die Übersättigung der Luft mit Kohlendioxyd drohen zu katastrophalen Klimaänderungen zu führen. Die afrikanische Sahelzone wird zur Wüste. Chemische Gifte, Lärm, Übermedikamentierung, soziale und sinnliche Frostigkeit schinden die bürgerlichen Körper und Seelen, die sich mit neuen Krankheiten und Neurosen (z.B. Krebs; Autismus) gegen diese Behandlung wehren. Das kapitalistische Verhältnis zur Natur schlägt sich im Umgang mit dem eigenen Körper nieder: er ist genauso entfremdet. Der bürgerliche Körper- und Gesundheitsbegriff sieht den Leib bloß als Instrument, das in der Produktion bis zum Zerreißen zergliedert und angespannt und nachher repariert wird.

Die innere und äußere Naturgrundlage des menschlichen Lebens, in Erinnerung und Sehnsucht, macht sich aber zunehmend, wie gestutzt auch immer, durch die bürgerlichen Lebensformen hindurch geltend. Sie ist anwesend in Naturphase und -rlebnis des Kindes, in der immerwährenden Sehnsucht nach Naturhaftem (welche die Werbung verwertet: wilde Frische von Limonen). Ebenso in der schillernden Bedeutung des Wortes ,natürlich" als ungekünstelt, anheimelnd, warm, aber auch als gefährlich und unberechenbar; das eine soll auch im bürgerlichen Leben bewahrt und ihm hinzugesetzt werden, das andere soll ausgesperrt werden. Die teilweise zu beobachtende künstliche Reproduktion zerschlagener Natur, gründet in jenem Widerspruch. Derartige späte Rettungsversuche gründen u.a. in der Erkenntnis der Bourgeoisie, der nun auch sie drückenden Umweltzerstörung - in natürlich möglichst profitabler Weise - Einhalt gebieten zu müssen. Selbst zu dieser reduzierten Erkenntnis freilich muß(te) sie erst noch gedrängt werden. Die künstliche Naturreproduktion bringt es, funktional und konsumorientiert, auf der selbstzerrissenen sozialen Grundlage der bestehenden Verhältnisse nur zu Zerrformen des Hingeschiedenen

27 Blüchel, a.a.O., S. 62 Ähnliche Äußerungen finden sich jedoch vielfach, vor allem in der neueren Welle von Umweltschutzbüchern, aber auch z.B. bei Konrad Lorenz.

28 Blüchel, a.2.O., S. 14

29 Vgl. Dieter Hassenpflug, Umweltzerstörung und Sozialkosten, Berlin (W) 1974; Herbert Gruhl, Ein Planet wird geplündert, Frankfurt/M 1978; Holger Strohm, Umweltschmutz, Darmstadt 1972; Juan Genet, Umweltschutz, Hamburg 1977 
(30), zur Park-, Stuyvesant-, Safari-, Regionalplanungs-, Lacknatur, bürgerliche Karikatur einer historisch möglichen Versöhnung von Mensch und Natur.

\section{$\mathrm{V}$}

Das Neue, ein verändertes Verhalten zur Natur, zeichnet sich ab. Das ,Erbe der Altmenschheit“, eine ,größere Lebensnähe und Lebenssicherheit“, der „Grundgedanke der Weltharmonie" (31), beginnt sich angesichts der drohenden Katastrophe und der neuen historischen Möglichkeiten zu regen.

Mit Castaneda z.B. hat das Verlangen, sich in die Natur und ein so verändertes Ich einzuschwingen, eine Bresche in das rationalistisch-instrumentalistisch verengte Weltbild geschlagen. Naturfreaks, Landkommunler, Wandervögel usw. sehen, daß der bürgerliche Weg nur zum Untergang der Natur führt. Sie ahnen die Befreiung zu einer nunmehr bewußt gesellschaftlichen Natur, die zur Mitproduktivität eingeholt ist und eigenes Subjekt wird. Aber sie neigen dazu, das isoliert zu tun, suchen die reine Natur, die es so schon lange nicht mehr und nie mehr gibt. Vom arbeitenden Austausch mit der Natur allein und unmittelbar läßt sich unter den derzeitigen Bedingungen, wo die industrielle Arbeit das Wertgesetz diktiert, nur sehr kärglich leben. Die Naturdebatte droht sich als Wiederholung der alten Emanzipationsdebatte zu entpuppen, wo alles letztlich nur auf die Rettung der kleinen eigenen Haut hinausläuft. Der Blick zurück in den Mythos repräsentiert eine ebenso alte wie ohnmächtige Kritik, wenn er seine utopische Dimension nicht hat, auf die sozialen Hebel zur Verweltlichung und Verwirklichung des Paradieses auf neuer, höherer Stufenleiter vor uns zu achten (32). Arabische Märchenerzähler, afrikanische Medizinmänner, Naturheilkundler allerorten', die Naturmystik in der modernen Frauenbewegung bewahren und beleben das alte Wissen um Natur und Mensch - und finden dabei in der hohen Wissenschaft zunehmend respektvolle Beachtung. Wirklich entfalten und weiterentwickeln können sie sich aber erst unter neuen gesellschaftlichen Umständen der Versöhnung des Menschen mit dem Menschen und mit der Natur.

Nachsinnen und Experimentieren zu einem neuen Naturverhältnis nehmen jedenfalls zu. So können Erfahrungen und Erkenntnisse gewonnen werden, wie die Natur nicht mehr zu würgen und zu beuteln, sondern pfleglich zu behandeln ist, wie Sinnlichkeit zu entwickeln und abstrakte Zeit(knappheit) zu überwinden ist, wie an die Stelle der Projektion der Eigencharakter der Natur dringen kann, wie die Abspaltung menschlicher Wesensqualitäten zurückgenommen werden kann, wie Denken und Sinnlichkeit vereinbart werden, wie das Verhältnis von Vernunft und Übersinnlichkeit aussieht - kurz wie die vielseitige reiche Persönlichkeit im Bund mit der Natur entwickelt werden kann.

30 Vgl. Horst Stern, a.a.O., S. $19 \mathrm{ff}$.

31 Werner Müller, a.a.O., S. 98 und 94

32 Das trifft etwa auf die moderne Indianerschwärmerei oder die Indienkreuzzüge zu. Só Werner Müller, a.a.O., S. 88: „Die Rolle des Westens als Befreier des Einzelnen von Magie und Mythos hat sich bitter gerächt." 
Im großen Maßstab ist ein solches Programm freilich nur auf der gesellschaftlichen Ebene zu realisieren, welche das ihm zugrundeliegende Problem bereits längst erreicht hat. Wenn die zivilisatorische Mission des Kapitalismus, den gesellschaftlichen Reichtum gewaltig zu entwickeln, die Naturgrundlagen des Lebens selbst zu vernichten und damit selbstzerstörerisch zu werden droht, sind Klassenfrage und Klassenfrage nicht obsolet, sondern erst eigentlich im emphatischen Sinne existentiell und emanzipativ-menschheitlich. Die Klasse, welche sich in ihrem Befreiungskampf um das Verhältnis von Natur und Gesellschaft nicht kümmert, hat ihre $\mathrm{Zu}$ kunft schon verspielt. Ein entwickeltes Naturbewußtsein ist eine Frage des politischen Klassenbewußtseins, der Aktion der Klasse gegen Kapital und Staat, um die Verpfuschung ihres jetzigen und zukünftigen Lebens zu verhindern.

Die Grundlage der Freiheit auf dem Gebiet der Naturbeherrschung und der Naturkräfte ,kann nur darin bestehen, daß der vergesellschaftete Mensch, die assoziierten Produzenten, diesen ihren Stoffwechsel mit der Natur rationell regeln, unter ihre gemeinschaftliche Kontrolle bringen, statt von ihm als einer blinden Macht beherrscht zu werden, ihn mit dem geringsten Kraftaufwand und unter den ihrer menschlichen Natur würdigsten und adäuatesten Bedingungen vollziehn" (33). Auf dieser Grundlage kann der Mensch in die neuen Räume einziehen, auf gleichberechtigter Basis mit der Natur verkehren und beider Wesenskräfte in ungeahntem Maße freisetzen. Ohne in Faselei zu verfallen, kann man annehmen, daß dies u.a. Schonung und Ehrfurcht, nichtherrscherliche Wißbegier und Lernbereitschaft gegenüber der Natur, Entwicklung und gute Übung der Sinne im Verein mit einer nicht mehr feindlichen Vernunft bedeuten wird. Eine neuere Tendenz im Science Fiction z.B. beinhaltet: „Der moderne Begriff der Natur als Objekt der Forschung und Kontrolle wird einer älteren Idee von der Natur als einem wundersamen Lebewesen, von dem der Mensch einen Teil bildet, untergeordnet" (33a). Hilflos und nach hinten wird hier das neue Recht der Natur ausgesprochen.

Ohne Widersprüche und Beschränkungen wird das nicht abgehen. „Nur dann, wenn klar zutage liegt, daß die Hoffnung auf Versöhnung von Natur und Geschichte schlicht unverschämt ist, solange man beides gleichermaßen will: durch totale Unterwerfung der sogenannten äußeren Natur luxurierende Menschheit und eine Natur, die die Augen aufschlägt, können überhaupt Lösungen in Sicht kommen “(34).

Bislang sind derartige Ansätze und Überlegungen weitgehend auf die Ökologieund Alternativbewegung beschränkt geblieben. Während sie auf gesellschaftlicher Ebene nur als-Klassenfrage wirklich angehbar sind, können Tendenzen in diese Richtung noch nicht ausgemacht werden. Eine Arbeiterbewegung, die weitgehend als gewerkschaftliche auftritt und in die bürgerliche Welt und Logik eingebettet ist, wie die westdeutsche, hat es sehr schwer, auf dem ökologischen Feld perspektivische und offensive klassenpolitische Lösungen zu entwickeln und durchzusetzen. Neuere Debatten und Aktionen um den Bau von Kernkraftwerken zeigen eher das Bild gewerkschaftlicher Spaltung und Entscheidungslosigkeit. So ist die Bewegung um

33 Kar1 Marx, Das Kapital, Bd. III, MEW 25, S. 828

33a Andrew Feenberg, An End To History, in: John Hopkins Magazine, March 1977

34 Reinhard Maurer, a.a.O., S. 138 
ein neues Verhältnis zur Natur vorerst auf wenige Köpfe und Randgruppen verwiesen. Die Naturbehandlung im realen Sozialismus ist auch wenig ermutigend. Häufig auf rückständiger materieller Basis, was bisweilen den Eindruck größerer Pfleglichkeit erweckt, gleicht sie doch im Grundsatz (möglichst schrankenlose Entwicklung der Produktivkräfte) dem Denken und Tun des bürgerlichen Industrialismus. Angesichts der Tiefe der notwendigen Veränderungen, die an den Kern der bürgerlichen Klassen- und Ichstruktur gehen, und angesichts der drohenden ökologischen Katastrophe sind die geșellschaftlich progressiven Antworten darauf noch unterentwickelt .

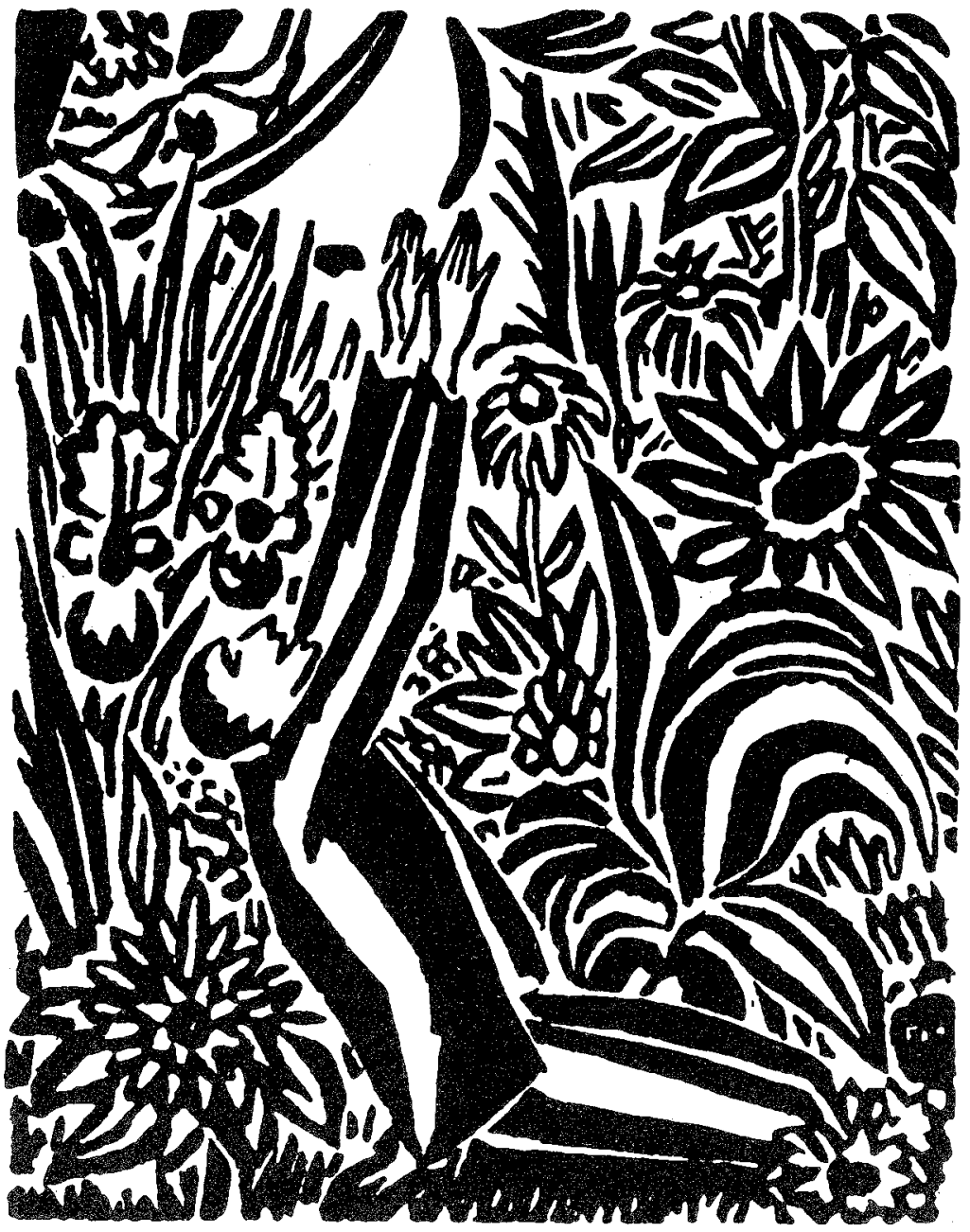

(aus: Frans Masereel, Mein Stundenbuch) 